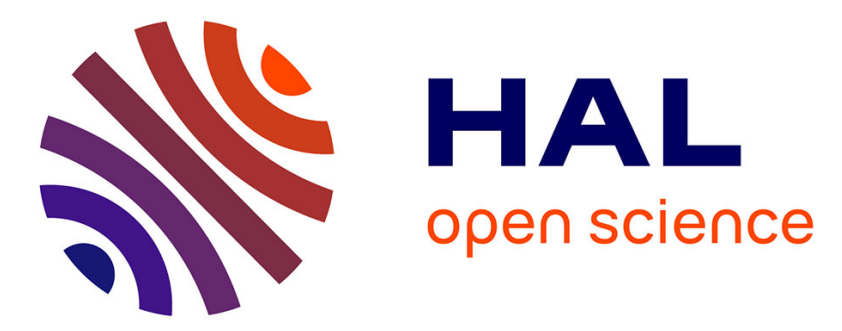

\title{
Incorporation of prior knowledge of the signal behavior into the reconstruction to accelerate the acquisition of MR diffusion data
}

\author{
Juan F P J Abascal, Manuel Desco, Juan Parra-Robles
}

\section{To cite this version:}

Juan F P J Abascal, Manuel Desco, Juan Parra-Robles. Incorporation of prior knowledge of the signal behavior into the reconstruction to accelerate the acquisition of MR diffusion data. IEEE Transactions on Medical Imaging, 2017, 10.1109/TMI.2017.2765281 . hal-01462812v2

HAL Id: hal-01462812

https://hal.science/hal-01462812v2

Submitted on 23 Oct 2017

HAL is a multi-disciplinary open access archive for the deposit and dissemination of scientific research documents, whether they are published or not. The documents may come from teaching and research institutions in France or abroad, or from public or private research centers.
L'archive ouverte pluridisciplinaire HAL, est destinée au dépôt et à la diffusion de documents scientifiques de niveau recherche, publiés ou non, émanant des établissements d'enseignement et de recherche français ou étrangers, des laboratoires publics ou privés. 


\title{
Incorporation of prior knowledge of signal behavior into the reconstruction to accelerate the acquisition of diffusion MRI data
}

\author{
Juan F. P. J. Abascal, Manuel Desco, and Juan Parra-Robles
}

\begin{abstract}
Diffusion MRI data are generally acquired using hyperpolarized gases during patient breath-hold, which yields a compromise between achievable image resolution, lung coverage and number of b-values. In this study, we propose a novel method that accelerates the acquisition of diffusion MRI data by undersampling in both the spatial and b-value dimensions and incorporating knowledge about signal decay into the reconstruction (SIDER). SIDER is compared with total variation (TV) reconstruction by assessing its effect on both the recovery of ventilation images and the estimated mean alveolar dimensions (MAD). Both methods are assessed by retrospectively undersampling diffusion datasets of healthy volunteers and COPD patients $(n=8)$ for acceleration factors between $x 2$ and $x 10$. TV led to large errors and artifacts for acceleration factors equal to or larger than $x 5$. SIDER improved $T V$, with a lower solution error and MAD histograms closer to those obtained from fully sampled data for acceleration factors up to $x 10$. SIDER preserved image quality at all acceleration factors, although images were slightly smoothed and some details were lost at $\mathbf{x 1 0}$. In conclusion, we developed and validated a novel compressed sensing method for lung MRI imaging and achieved high acceleration factors, which can be used to increase the amount of data acquired during breath-hold. This methodology is expected to improve the accuracy of estimated lung microstructure dimensions and provide more options in the study of lung diseases with MRI.
\end{abstract}

Index Terms-Compressed sensing, lung diffusion MRI, hyperpolarized gas MRI, sparsity, split Bregman method

\section{INTRODUCTION}

$T$ HE increase in MRI sensitivity using hyperpolarized contrast media has enabled the development of imaging techniques to assess anatomical features and functional processes beyond the limits of conventional MRI [1], [2]. In particular, hyperpolarized noble gas MRI can provide quantitative maps of clinically relevant anatomical and physiological parameters (e.g. ventilation distribution [3], [4], acinar airway dimensions [5], alveolar oxygen partial pressure $\left(\mathrm{pO}_{2}\right)$ [6], [7], and gas washout [8]). An increase in the MRI signal is achieved using laser polarization techniques (e.g. optical pumping-spin exchange [9], [10]), which result in a nonequilibrium net magnetization that is up to 5 orders of magnitude higher than in conventional MRI (thermally polarized).

However, the non-renewable nature of the magnetization in hyperpolarized gases imposes limits on the duration of MRI acquisition. In the presence of oxygen, the $\mathrm{T} 1$ of hyperpolarized gases (15-20 s) is of the order of duration of the breath-hold that can be achieved by patients within the scanner. As a consequence of this limitation, together with the high cost of the gas (e.g. ${ }^{4} \mathrm{He}$ and isotopically enriched ${ }^{129} \mathrm{Xe}$ ), most hyperpolarized gas methods aim to perform a complete acquisition during a single breath-hold using a single hyperpolarized gas dose. Furthermore, there are advantages in acquisition with more than one sequence or even different nuclei during a single breath-hold [11]. This need for rapid acquisition has been addressed using various accelerated

J.F.P.J. Abascal was with the Departamento de Bioingeniería e Ingeniería Aeroespacial, Universidad Carlos III de Madrid (UC3M), 28903, Leganes, Spain and with Instituto de Investigación Sanitaria Gregorio Marañón (IiSGM), 28007 Madrid, Spain, and is now with Universite de Lyon, INSA-Lyon, Université Claude Bernard Lyon 1, UJM-Saint Etienne, CNRS, Inserm, CREATIS UMR 5220, U1206, F-69100, Lyon, France (email: juan.abascal@creatis.insa-lyon.fr).

M. Desco and J. Parra-Robles are with Departamento de Bioingeniería e Ingeniería Aeroespacial, Universidad Carlos III de Madrid (UC3M), 28903 Leganes, Spain and with Instituto de Investigación Sanitaria Gregorio Marañón (IiSGM), 28007 Madrid, Spain (email: desco@hggm.es), (email: juparrar@ing.uc3m.es).

M. Desco is also with Centro de Investigaciones Cardiovasculares Carlos III (CNIC) and with Centro de Investigación en Red de Salud Mental (CIBERSAM), 28029 Madrid, Spain. com the European Union's Seventh Framework Program (FP7/2007-2013) and EFPIA companies' in kind contribution. This project has received funding from the European Union's Horizon 2020 research and innovation programme under the Marie Sklodowska-Curie grant agreement $\mathrm{N}^{\circ}$ 701915 .

(C) 20xx IEEE. Personal use of this material is permitted. Permission from IEEE must be obtained for all other uses, in any current or future media, including reprinting/republishing this material for advertising or promotional purposes, creating new collective works, for resale or redistribution to servers or lists, or reuse of any copyrighted component of this work in other works. DOI 10.1109/TMI.2017.2765281 
This article has been accepted for publication in a future issue of this journal, but has not been fully edited. Content may change prior to final publication. Citation information: DOI 10.1109/TMI.2017.2765281, IEEE Transactions on Medical Imaging

acquisition approaches, including parallel imaging [12], [13] and compressed sensing (CS) [14], [15]. In CS, the acquisition was accelerated in the spatial encoding direction and the images were reconstructed using spatial total variation (TV). More recently, Chan et al [16] used CS to acquire diffusion images of hyperpolarized gases in the lungs. Diffusion MRI images are sensitive to changes in lung microstructure [17] due to disease and are used to estimate the dimensions of acinar airways based on theoretical models obtained from numerical simulations. These theoretical models require the acquisition of images for several diffusion sensitization values (b-values), which, together with the longer duration of the diffusion scan (due to the presence of diffusion gradients), result in long acquisition times. Owing to the limitation imposed by breath-hold duration, there is a compromise between achievable image resolution, number of slices and number of b-values, which limits the accuracy and number of parameters of the theoretical models [18]. Typically, most protocols acquire 5 slices $(10 \mathrm{~mm}$ thick, spacing $10 \mathrm{~mm}$ ) with a resolution of $64 \times 64$ pixels and 4 to $6 \mathrm{~b}$ values, thus leading lung coverage to be sacrificed [19]. Chan et al. achieved full lung coverage by using CS with a 3D diffusion acquisition and undersampling along the two phaseencoded spatial directions [16]. CS has also been proposed for accelerating diffusion-weighted imaging [5], [20]-[22]. In diffusion spectrum imaging, CS has been applied using adaptive dictionaries [23] and different types of undersampling patterns and sparsifying transforms [24], [25].

Although ${ }^{3} \mathrm{He}$ lung diffusion images are sparser in the bdirection than in the spatial domain, the feasibility of exploiting sparsity along both the spatial encoding and the b-value directions has not been studied in hyperpolarized gas MRI. In other MRI applications, such as in cardiac cine MRI [26]-[28] and fMRI [29], [30], CS leads to large acceleration factors by exploiting high data dimensionality.

In this study, we propose a novel CS method that incorporates a model of the signal decay as prior information into the reconstruction (SIDER) to accelerate the acquisition of diffusion-weighted MRI data by undersampling in both the spatial and the $b$-value dimensions. We incorporate knowledge of diffusion signal behavior into the reconstruction to accelerate the acquisition of diffusion MRI data. The method we propose is compared with TV and zero filling reconstructions by assessing its effect on the estimated parameters of a stretched exponential model, which has been used to estimate mean alveolar dimensions (MAD) [31], [32]. The methods were applied in control and COPD patient data sets ( $\mathrm{n}=8$ in total) using retrospective undersampling simulations, with fully sampled data as the gold standard.

\footnotetext{
${ }^{1}$ Results and MATLAB code for TV and SIDER methods are available from the GitHub repository https://github.com/HGGM-LIM/compressed-sensingdiffusion-lung-MRI
}

\section{METHODS}

\section{A. Image reconstruction methods}

\section{1) Total variation}

Previous CS studies for MRI using hyperpolarized gases assumed that each ventilation image $u_{i}$ is sparse under a transformation $\Psi$, which accounts for spatial sparsity [14]-[16]. The most common choice for $\Psi$ is the gradient that leads to the total variation functional. If $F$ represents the undersampled Fourier transform and $f_{i}$ represents the undersampled k-space corresponding to the $i$-th $\mathrm{b}$-value, then the total variation problem is given by

$$
\min _{u_{i}}\left\|\nabla u_{i}\right\|_{1} \text { such that }\left\|F u_{i}-f_{i}\right\|^{2} \leq \sigma^{2},
$$

where $\nabla=\left(\nabla_{x}, \nabla_{y}\right), i=1, \ldots, B$, and $B$ is the total number of $\mathrm{b}$ values.

\section{2) Signal decay-based reconstruction}

We propose a novel CS method based on undersampling in both the spatial and the $b$-value dimensions that incorporates a model of the signal decay into the reconstruction (SIDER). It combines TV with a penalty function that promotes sparsity across the b-direction as follows:

$$
\min _{u} \alpha\|\nabla u\|_{1}+\beta\|M u\|_{1} \text { such that }\|F u-f\|^{2} \leq \sigma^{2},
$$

where $u$ and $f$ are the ventilation images and undersampled data corresponding to all values of $b, u=\left[u_{1}{ }^{\mathrm{H}}, \ldots, u_{B}{ }^{\mathrm{H}}\right]^{\mathrm{H}}, f=\left[f_{1}^{\mathrm{H}}, \ldots, f\right.$ $\left.{ }_{B}{ }^{\mathrm{H}}\right]^{\mathrm{H}}, u^{\mathrm{H}}$ denotes the Hermitian transpose of $u, F$ is the undersampled multislice Fourier transform, and $M$ is an operator that encodes the relationship between ventilation images for consecutive values of $b$. This relationship can be approximated using a stretched exponential model [16], [31] as

$$
M u_{j}=u_{j}-u_{j-1} \exp \left[-\left(\left(\bar{D} b_{j}\right)^{\bar{\alpha}}-\left(\bar{D} b_{j-1}\right)^{\bar{\alpha}}\right)\right],
$$

where $u_{j}$ is the ventilation image for the $j$-th $b$-value and $\bar{D}$ and $\bar{\alpha}$ are the estimated average value of diffusivity and the heterogeneity index, respectively, across the whole volume, which can be obtained from a previously reconstructed image (here we used the image provided by the TV method). ${ }^{1}$ This global estimation for the model parameter provides sufficient a priori information to guide the image reconstruction. The transform $M$ is a generalization of the gradient across the $b$ dimension, which takes the signal decay into account. Ventilation images are actually sparser on the stretched exponential domain defined by $M$ than under the spatial gradient domain.

\section{3) Split Bregman formulation}

The problems in (1) and (2) were solved using the Split Bregman formulation, which efficiently handles L1-based constrained problems [27], [28], [33], [34]. Using this formulation, constrained problems are converted to equivalent unconstrained problems, where constraints are imposed

(C) 20xx IEEE. Personal use of this material is permitted. Permission from IEEE must be obtained for all other uses, in any current or future media, including reprinting/republishing this material for advertising or promotional purposes, creating new collective works, for resale or redistribution to servers or lists, or reuse of any copyrighted component of this work in other works. DOI 10.1109/TMI.2017.2765281 
This article has been accepted for publication in a future issue of this journal, but has not been fully edited. Content may change prior to final publication. Citation information: DOI 10.1109/TMI.2017.2765281, IEEE Transactions on Medical Imaging

iteratively using the Bregman iteration. L2- and L1-norm functionals are separated into several subproblems, which are solved analytically in alternating steps. The subproblem including the L2-norm functionals results in a linear system that can be efficiently solved using iterative Krylov solvers; subproblems including L1-norm functionals are solved using shrinkage formulas. As TV can be obtained from SIDER by making $\beta=0$, we develop the formulation for the general case of SIDER.

To perform the split, we include the new variables $d_{x}, d_{y}$, and $w$ and formulate a new problem that is equivalent to (2):

$$
\begin{aligned}
& \min _{u, d_{x}, d_{y}, w} \alpha\left\|\left(d_{x}, d_{y}\right)\right\|_{1}+\beta\|w\|_{1} \text { such that }\|F u-f\|^{2} \leq \sigma^{2}, \\
& d_{x}=\nabla_{x} u, \quad d_{y}=\nabla_{y} u, \quad w=M u
\end{aligned}
$$

Equation (4) is now easily managed using an equivalent unconstrained optimization approach, in which constraints are imposed by adding Bregman iterations $b_{i}$,

$$
\begin{aligned}
& \min _{u, d_{x}, d_{y}, w} \alpha\left\|\left(d_{x}, d_{y}\right)\right\|_{1}+\beta\|w\|_{1}+\frac{\mu}{2}\left\|F u-f^{k}\right\|_{2}^{2}+ \\
& \frac{\lambda}{2}\left\|d_{x}-\nabla_{x} u-b_{x}^{k}\right\|_{2}^{2}+\frac{\lambda}{2}\left\|d_{y}-\nabla_{y} u-b_{y}^{k}\right\|_{2}^{2}+\frac{\lambda}{2}\left\|w-M u-b_{w}^{k}\right\|_{2}^{2}
\end{aligned}
$$

where $\mu$ is a parameter that weights the data fidelity term, $\lambda$ is another parameter that weights the terms imposing the constraints for the dummy variables, $k$ is the iteration number and the Bregman iterations are updated as

$$
\begin{gathered}
b_{x}{ }^{k+1}=b_{x}{ }^{k}+\nabla_{x} u^{k+1}-d_{x}{ }^{k+1} \\
b_{y}{ }^{k+1}=b_{y}{ }^{k}+\nabla_{y} u^{k+1}-d_{y}{ }^{k+1} \\
b_{w}{ }^{k+1}=b_{w}{ }^{k}+M u^{k+1}-w^{k+1} \\
f^{k+1}=f^{k}+f-F u^{k+1}
\end{gathered}
$$

Since $u$ and auxiliary variables $w, d_{x}$, and $d_{y}$ are independent of each other, equation (5) can now be split into several equations (one for each variable) that are solved sequentially, as follows:

$$
\begin{aligned}
& u^{k+1}=\underset{u}{\arg \min } \frac{\mu}{2}\left\|F u-f^{k}\right\|_{2}^{2}+\frac{\lambda}{2}\left\|d_{x}{ }^{k}-D_{x} u-b_{x}^{k}\right\|_{2}^{2}+ \\
& +\frac{\lambda}{2}\left\|d_{y}{ }^{k}-D_{y} u-b_{y}^{k}\right\|_{2}^{2}+\frac{\lambda}{2}\left\|w^{k}-M u-b_{w}^{k}\right\|_{2}^{2} \\
& \left(d_{x}{ }^{k+1}, d_{y}{ }^{k+1}\right)=\underset{d_{x}, d_{y}}{\arg \min } \alpha\left\|\left(d_{x}, d_{y}\right)\right\|_{1}+\frac{\lambda}{2}\left\|d_{x}-D_{x} u^{k+1}-b_{x}^{k}\right\|_{2}^{2}+ \\
& +\frac{\lambda}{2}\left\|d_{y}-D_{y} u^{k+1}-b_{y}^{k}\right\|_{2}^{2} \\
& w^{k+1}=\underset{w}{\arg \min } \beta\|w\|_{1}+\frac{\lambda}{2}\left\|w-M u^{k+1}-b_{w}^{k}\right\|_{2}^{2}
\end{aligned}
$$

Since the solution of $u$ only involves L2-norm functionals, it can be obtained exactly as the solution of the linear system

$$
\begin{aligned}
K u^{k+1}=r^{k} & \\
K & =\mu F^{\mathrm{H}} F+\lambda D_{x}{ }^{\mathrm{H}} D_{x}+\lambda D_{y}{ }^{\mathrm{H}} D_{y}+\lambda M^{\mathrm{H}} M \\
r^{k} & =\mu F^{\mathrm{H}} f^{k}+\lambda D_{x}{ }^{\mathrm{H}}\left(d_{x}{ }^{k}-b_{x}^{k}\right)+\lambda D_{y}{ }^{\mathrm{H}}\left(d_{y}{ }^{k}-b_{y}{ }^{k}\right) \\
& +\lambda M^{\mathrm{H}}\left(w^{k}-b_{w}{ }^{k}\right)
\end{aligned}
$$

where $w^{\mathrm{H}}$ denotes the Hermitian transpose of $w$. It is common practice to modify (7) by adding a quadratic term to improve the stability of the algorithm [34]. This replaces the update for $u^{k+1}(8)$ as follows:

$$
\begin{aligned}
& K u^{k+1}=r^{k} \\
& K=\mu F^{\mathrm{H}} F+\lambda D_{x}{ }^{\mathrm{H}} D_{x}+\lambda D_{y}{ }^{\mathrm{H}} D_{y}+\lambda M^{\mathrm{H}} M+\gamma I \\
& r^{k}=\mu F^{\mathrm{H}} f^{k}+\lambda D_{x}{ }^{\mathrm{H}}\left(d_{x}{ }^{k}-b_{x}{ }^{k}\right)+\lambda D_{y}{ }^{\mathrm{H}}\left(d_{y}{ }^{k}-b_{y}{ }^{k}\right) \\
& \\
& \quad+\lambda M^{\mathrm{H}}\left(w^{k}-b_{w}{ }^{k}\right)+\gamma u^{k}
\end{aligned}
$$

Note that (9) constitutes a very large-scale problem, where $\mathrm{K}=\mathrm{NxN}$ and $\mathrm{N}$ is the number of pixels, yet it can be solved efficiently using a Krylov solver, such as the biconjugate gradient stabilized method, which involves only matrix-vector multiplications:

$$
\begin{aligned}
r^{k}= & \mu F^{H}(F u)+\lambda D_{x}^{H}\left(D_{x} u\right)+\lambda D_{y}{ }^{H}\left(D_{y} u\right)+ \\
& +\lambda M^{H}(M u)+\gamma u
\end{aligned}
$$

The auxiliary variables $d_{x}, d_{y}$, and $w$ are solved analytically using shrinkage formulas, which are thresholding operations $(1,2)$

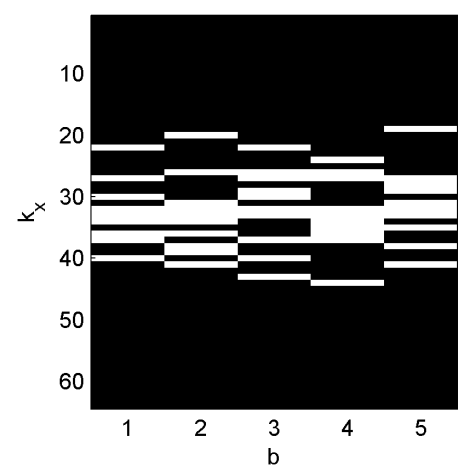

Fig. 1. Undersampling pattern for an acceleration factor of $\mathrm{x} 7$. Randomization is performed in the phase-encoding direction and through the b-direction, for a $64 \times 64$ resolution and $5 \mathrm{~b}$-values.

$$
\begin{aligned}
& d_{j}^{k+1}=\max \left(s^{k}-\alpha / \lambda, 0\right) \frac{\left|D_{j} u^{k+1}+b_{j}^{k}\right|}{s^{k}}, j=x, y \\
& s^{k}=\sqrt{\left|D_{x} u^{k+1}+b_{x}^{k}\right|^{2}+\left|D_{y} u^{k+1}+b_{y}^{k}\right|^{2}}, \\
& w^{k+1}=\operatorname{shrink}\left(M u^{k+1}+b_{w}^{k}, \beta / \lambda\right)= \\
& =\max \left(\left|M u^{k+1}+b_{w}^{k}\right|-\beta / \lambda, 0\right) \operatorname{sign}\left(M u^{k+1}+b_{w}^{k}\right)
\end{aligned}
$$

\section{B. Datasets and retrospective undersampling}

Eight fully sampled diffusion datasets were available from a previous study, 3 from healthy volunteers [19] and 5 from three patients with COPD (2 patients had 2 acquisitions at different sessions) [31]. Data consisted of 5 slices $(10 \mathrm{~mm}$ thick with 10 $\mathrm{mm}$ gap between slices), $64 \times 64$ resolution and $5 \mathrm{~b}$-values ( 0 , $1.6,3.2,4.8$ and $6.4 \mathrm{~s} / \mathrm{cm}^{2}$ ), obtained with a diffusion time of $1.6 \mathrm{~ms}$. These data were acquired in a GE HDx $1.5 \mathrm{~T}$ scanner (GE Healthcare, USA) using a 2D spoiled gradient echo sequence with bipolar diffusion sensitization gradients [19],

(C) 20xx IEEE. Personal use of this material is permitted. Permission from IEEE must be obtained for all other uses, in any current or future media, including reprinting/republishing this material for advertising or promotional purposes, creating new collective works, for resale or redistribution to servers or lists, or reuse of any copyrighted component of this work in other works. DOI 10.1109/TMI.2017.2765281 
This article has been accepted for publication in a future issue of this journal, but has not been fully edited. Content may change prior to final publication. Citation information: DOI 10.1109/TMI.2017.2765281, IEEE Transactions on Medical Imaging

[31]. ${ }^{3} \mathrm{He}$ gas was polarized using a SEOP commercial polarizer (Helispin, GE Healthcare, USA), which achieved polarizations of $30-40 \%$.

These datasets were retrospectively undersampled to simulate CS acquisition and reconstruction. Quasi-random undersampling patterns were created using a variable density pattern as proposed in [35], [36]. The patterns were based on the polynomial probability density function of the form $(1-r)^{p}$, which assigned the highest probability to the center of the $k$ space and then assigned probabilities with a decay rate of $p$ as a function to the center. Randomization was performed in the phase-encoding direction and through the b-direction (Fig. 1), meaningful. (The upper limits for these parameters correspond to diffusion within structures much larger than acinar airspaces, i.e. free diffusion with $D_{0}=0.87 \mathrm{~cm}^{2} / \mathrm{s}$ and $\alpha=1$ ). Areas of the image that were outside this range appear black on the estimated maps. The process for estimating $\mathrm{L}_{\mathrm{m}}$ from ventilation images, $u(b)$, as well as the differences between the patient and control data sets are shown in Fig. 2.

All retrospectively undersampled data sets $(n=8$ for acceleration factors $\mathrm{x} 2, \mathrm{x} 4, \mathrm{x} 5, \mathrm{x} 7$, and $\mathrm{x} 10$ ) were reconstructed with zero filling (ZF), TV and SIDER. These methods were evaluated in terms of the following metrics: 1) relative meansquare error (MSE) of the recovered ventilation images, 2)

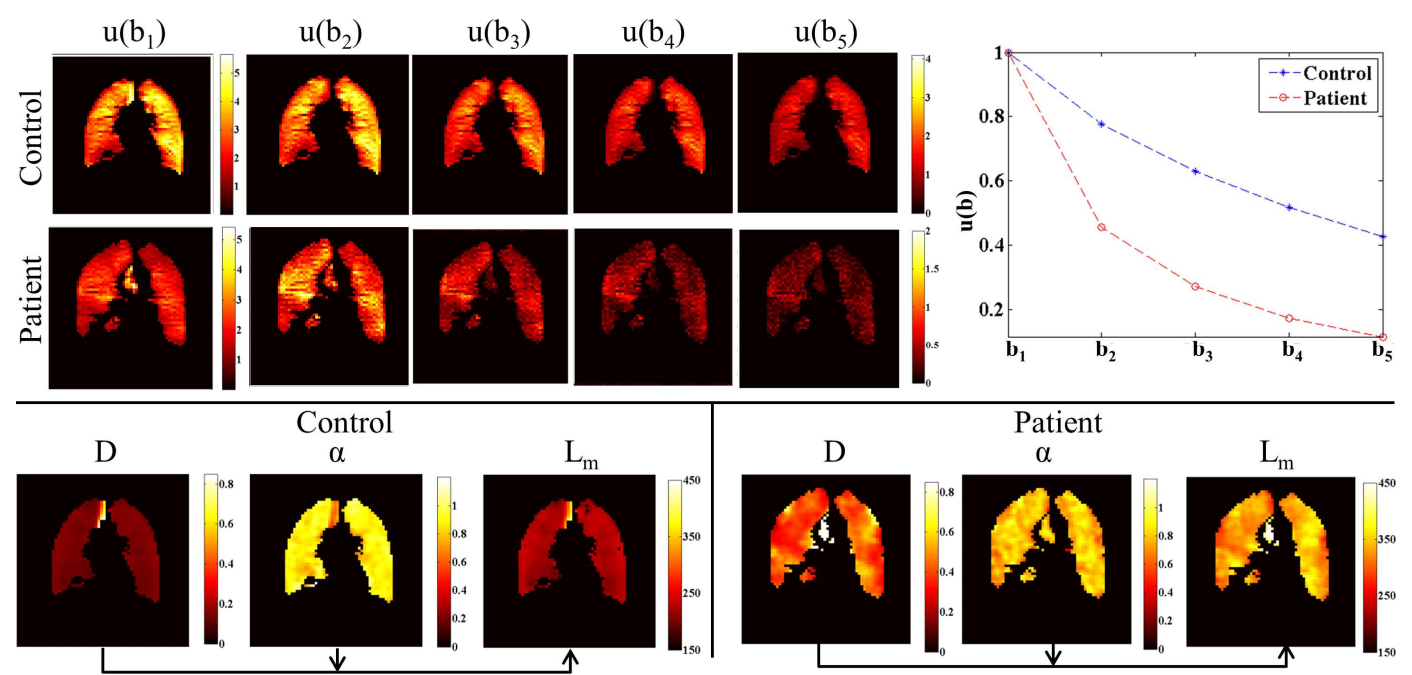

Fig. 2. Process for estimating mean alveolar dimensions, $\mathrm{L}_{\mathrm{m}}$, from ventilation images, $u(b)$, for datasets from a control and COPD patient. Top left: slice of ventilation images for different values of $b$. Top right: mean value of $u(b)$ across the slice to illustrate the typical difference in signal decay between controls and patients. Bottom: Estimated maps of distributed diffusion coefficient $D$, heterogeneity index $\alpha(12)$ and $\mathrm{L}_{\mathrm{m}}$ for one control and one patient.

thus enabling us to exploit data redundancy in 2 dimensions. We analyzed the results for acceleration factors of $\mathrm{x} 2, \mathrm{x} 4, \mathrm{x} 5$, $\mathrm{x} 7$, and $\mathrm{x} 10$. The decay rate was set to $p=4$ (for $\mathrm{x} 2$ ), $p=4$ (for $\mathrm{x} 4), p=4$ (for $\mathrm{x} 5$ ), $p=6$ (for $\mathrm{x} 7$ ) and $p=9$ (for $\mathrm{x} 10$ ).

\section{Image analysis and evaluation}

To evaluate the results, we first fitted the reconstructed signal $u(b)$ on a pixel-by-pixel basis to the stretched exponential model, which estimates maps of the distributed diffusion coefficient $D$ and heterogeneity index $\alpha$ [31]:

$$
u(b)=u(0) \exp \left(-(b D)^{\alpha}\right)
$$

As reconstructed images $u(b)$ are noisy, especially for patients and larger values of $\mathrm{b}$, images $u(b)$ were smoothed using a Gaussian filter (window of 3 neighboring pixels and SD of 1 pixel) before fitting the model in (12). $D$ and $\alpha$ were estimated only within a mask that had been created by segmenting the lungs in ventilation images from fully sampled data. Then, we estimated the $\mathrm{MAD}, \mathrm{L}_{\mathrm{m}}$, from $D$ and $\alpha$ as described in [16], [32]. $\mathrm{L}_{\mathrm{m}}$ was estimated only in the ranges $0<D<0.9$ and $0.3<\alpha<1$, which were assumed to be physically relative MSE of MAD, 3) histograms and 4) mean values of the estimated maps of MAD. MSE was computed by adopting the images and maps obtained from the fully sampled data as the gold standard. The results are given as mean and SD across all data sets. To assess the statistical significance of the difference between the methods, we used a Mann-Whitney test, which is robust and avoids the assumption of normality in the data.

Histograms and images are shown for 1 control and 1 patient data set for all methods. The mean and $\mathrm{SE}$ of $\mathrm{L}_{\mathrm{m}}$ in a region of interest ( 1 slice) are shown for the 3 patients to verify that errors due to undersampling were smaller than patient variability.

To determine the maximum acceleration factor achieved by the SIDER method, we studied its ability to discriminate between patients and controls and compared it with the reproducibility of the experiment. COPD can be diagnosed in terms of the histogram of $\mathrm{L}_{\mathrm{m}}$, as patients present larger values of $\mathrm{L}_{\mathrm{m}}$ and a larger spread of the histogram. We computed the percentage error of the estimated mean of $\mathrm{L}_{\mathrm{m}}$ as a function of the acceleration factor and the percentage error of the crosscorrelation between the histogram corresponding to each acceleration factor and that of the histogram of the complete data set. The reproducibility of the experiment was estimated based on 2 patients who had undergone the experiment twice the same day. Images and histograms for all datasets are shown

(C) 20xx IEEE. Personal use of this material is permitted. Permission from IEEE must be obtained for all other uses, in any current or future media, including reprinting/republishing this material for advertising or promotional purposes, creating new collective works, for resale or redistribution to servers or lists, or reuse of any copyrighted component of this work in other works. DOI 10.1109/TMI.2017.2765281 
This article has been accepted for publication in a future issue of this journal, but has not been fully edited. Content may change prior to final publication. Citation information: DOI 10.1109/TMI.2017.2765281, IEEE Transactions on Medical Imaging

for the SIDER method and for the maximum acceleration factor.

To validate the feasibility of SIDER for differentiation between control and COPD patients, we run a Mann-Whitney test to assess statistical significance between control and patients and between SIDER and fully sampled data.

\section{RESULTS}

\section{1) Selection of the reconstruction algorithm parameters}

The parameters necessary for the TV and SIDER methods are $\mu, \lambda, \gamma$, and the number of iterations. The parameter $\mu$ that weights the data fidelity term was selected following suggestions from previous studies [28], [33], [34], [36]-[38], which showed that the algorithm converges to the same solution independently of its value, as long as it is sufficiently small. In [37], a two-fold decrease in $\mu$ doubled the number of iterations $\lambda>20$, the convergence became unstable. Values of $\lambda<1$ resulted in low smoothing at the first iterations and an excessively high threshold in the shrinkage formulas, which also led to unstable convergence and large errors. Values in the range $1 \leq \lambda \leq 20$ led to similar results. The parameter $\gamma$ makes it possible to keep the Hessian matrix $K(9)$ well-conditioned when $\mu$ is relatively larger than $\lambda$. A small value of $\gamma$ is usually chosen $\left(10^{-4} \leq \gamma \leq 10^{-}\right.$ $\left.{ }^{2}\right)$.

The weighting parameters that control the relative degree of sparsity between TV $(\alpha)$ and model decay sparsity ( $\beta$ ) were heuristically determined as follows. Increasing $\alpha>1$ (higher sparsity to TV) led to images largely affected by cartoon-like artifacts and large solution errors. Selecting $\alpha<0.1$ led to excessively fast convergence, compromising the robustness of the algorithm. Values of $\alpha$ in the range $0.1<\alpha<1$ suppressed
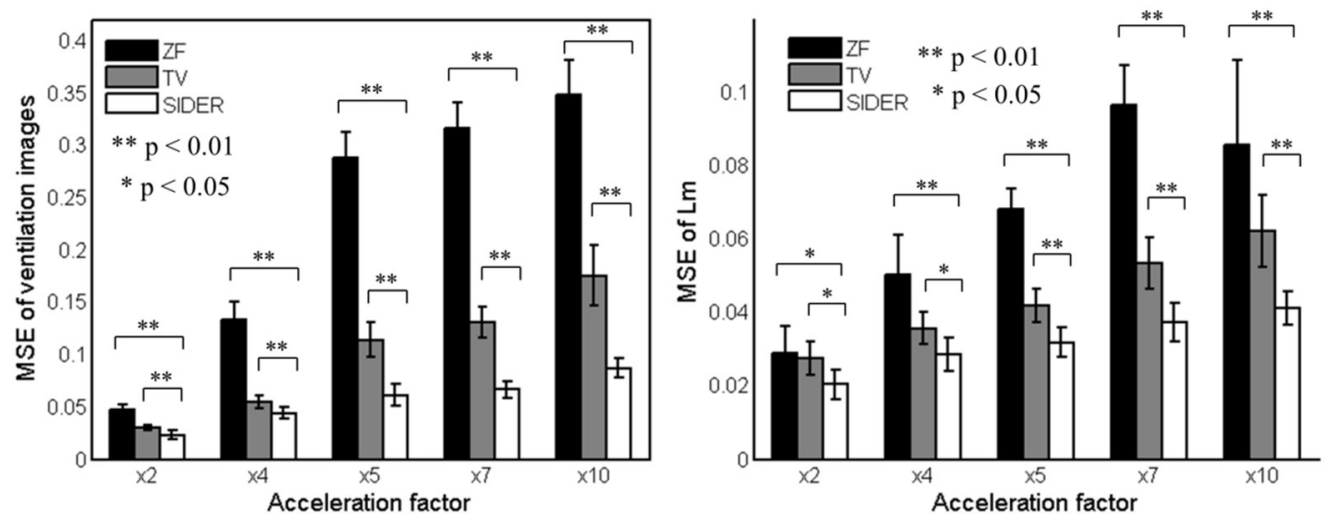

Fig. 3. MSE of ventilation images for $b=0$ (left) and estimated mean alveolar dimension (right) versus the acceleration factor for the different reconstruction algorithms. Results show mean and SD across all data sets $(n=8)$.

needed to converge to the same solution. Hence, the larger the value of $\mu$, the faster the convergence, although if $\mu$ is excessively large, the algorithm converges to a noisy solution on the first iterations. In contrast to unconstrained optimization, where optimal selection of the regularization parameters is needed based on the L-curve or a similar method [39], [40], we only need to select the number of iterations (stopping criterion).

TABLE I

REGULARIZATION PARAMETERS FOR TV AND SIDER

\begin{tabular}{lccccc}
\hline \hline & $\alpha$ & $\beta$ & $\mu$ & $\lambda$ & $\gamma$ \\
\hline TV & 1 & - & 1 & 1 & 1 \\
SIDER & 0.2 & 0.2 & 1 & 1 & 0.01 \\
\hline \hline
\end{tabular}

The stopping criterion used in previous studies was the discrepancy principle [34] or a fixed number of iterations [28]. In order to compare TV and SIDER in this study, the number of iterations was chosen to minimize the MSE, considering the fully sampled image as the correct solution. For $\mu \leq 2$, the method converged to the same solution at different iteration numbers. For $\mu>2$ overfitting occurred at the first iterations and an optimal solution was not found. Increasing $\lambda$ (higher weight to the TV constraint) lowers the threshold in the shrinkage formulas (11), resulting in slightly faster convergence, but for most noise in the image and yielded similar results. Increasing $\beta$ imposed higher sparsity on the model of signal decay than on $\mathrm{TV}$, leading to lower solution errors, images with fewer cartoon-like artifacts and images less affected by noise (especially images corresponding to large b-values). However, the larger the value of $\beta$, the slower the convergence. We found that values in the range $0.2 \leq \beta \leq 1$ were a good compromise. Table 1 shows a summary of the parameter values used for both $\mathrm{TV}$ and SIDER. In order to use the same range of parameters for all data sets, the algorithm normalizes the data item $f$ as $f\|f / n\|$, where $n$ is the square root of the number of pixels in the image, following the suggestions from [Tom Goldstein. Split Bregman. Retrieved in 2009 from http://www.ece.rice.edu/ tag7/Tom_Goldstein/Split_Bregman .html].

\section{B. Comparison of methods}

Fig. 3 (left) shows the MSE of the reconstruction of ventilation images (for $b=0$ ) with the various methods for all the acceleration factors tested. SIDER led to a significantly lower MSE than ZF and TV in all cases (for $b=0$ ); SIDER also led to a lower MSE for large values of $b$, although the differences were significant only at high accelerations. 
This article has been accepted for publication in a future issue of this journal, but has not been fully edited. Content may change prior to final publication. Citation information: DOI 10.1109/TMI.2017.2765281, IEEE Transactions on Medical Imaging

With an MSE of $10 \%$ as a reference for the comparison, the acceleration factors achieved by the methods were $\mathrm{x} 2$ for $\mathrm{ZF}$, and for the highest acceleration factors $(\mathrm{x} 5, \mathrm{x} 7$, and $\mathrm{x} 10)$ are shown in Fig. 4 for one control dataset and one patient dataset,

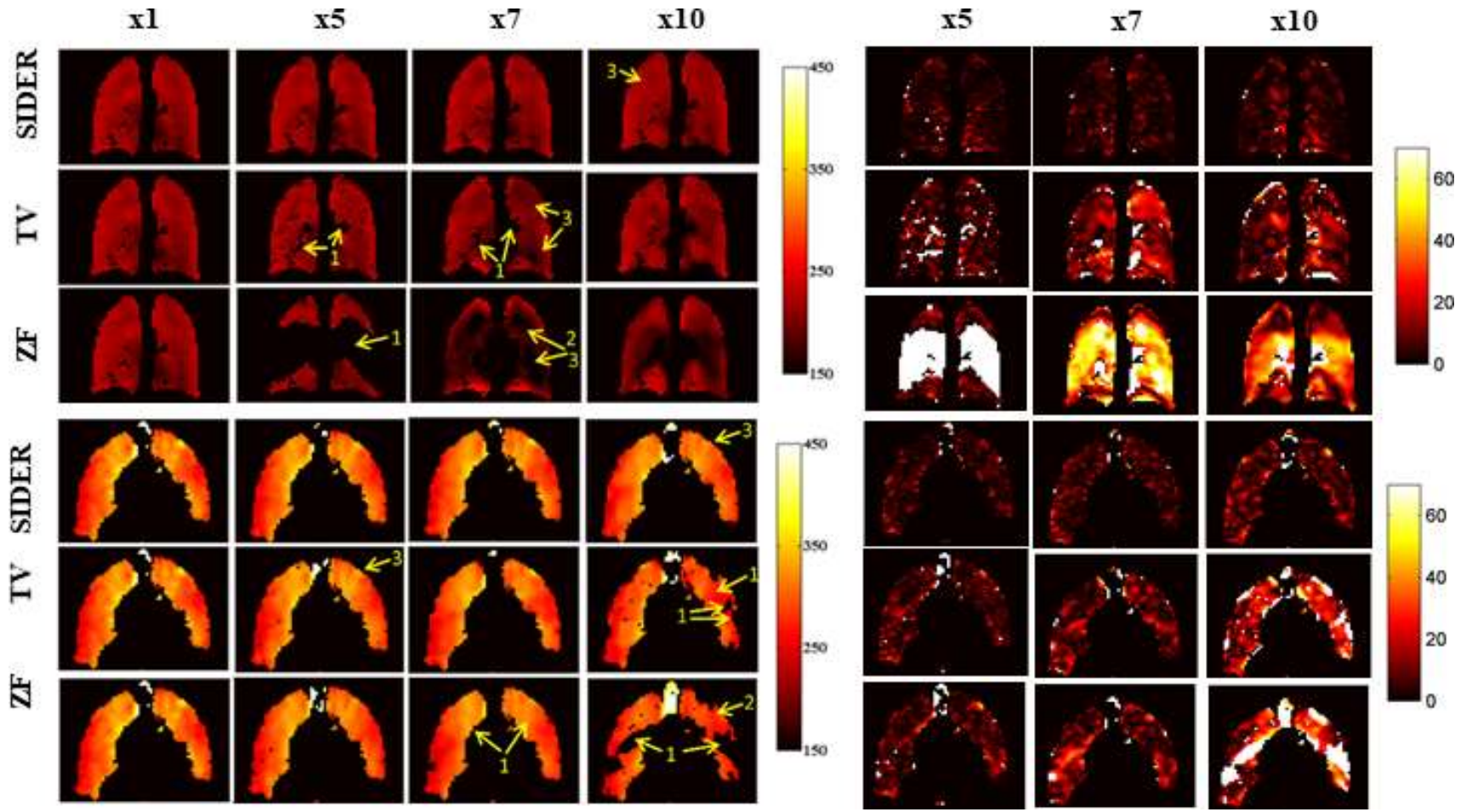

Fig 4. One slice of an estimated mean alveolar length map (left) and image differences (right) for each of the methods and acceleration factors, for a control subject (top) and for a COPD patient (bottom). The arrows point to areas where errors are more visible: (1) Black regions where estimated values were outside what is physically reliable, (2) shift in the mean values (3) and other errors in the estimated maps.

$\mathrm{x} 5$ for TV and $\mathrm{x} 10$ for SIDER. SIDER also presented a significantly lower MSE of MAD for all acceleration factors (Fig. 3, right). Similar to the MSE of ventilation images, at x10, SIDER presented the same MSE as TV at $\mathrm{x} 5$.

Maps of MAD and image differences for the various methods respectively ${ }^{2}$. In the case of the control dataset, for acceleration factor x5, ZF and TV led to errors and artifacts, whereas SIDER provided maps that were almost identical to the fully sampled dataset. For acceleration factor $\mathrm{x} 10, \mathrm{ZF}$ and TV led to larger errors and artifacts and a shift in the mean value. In contrast,
$\mathrm{ZF}$
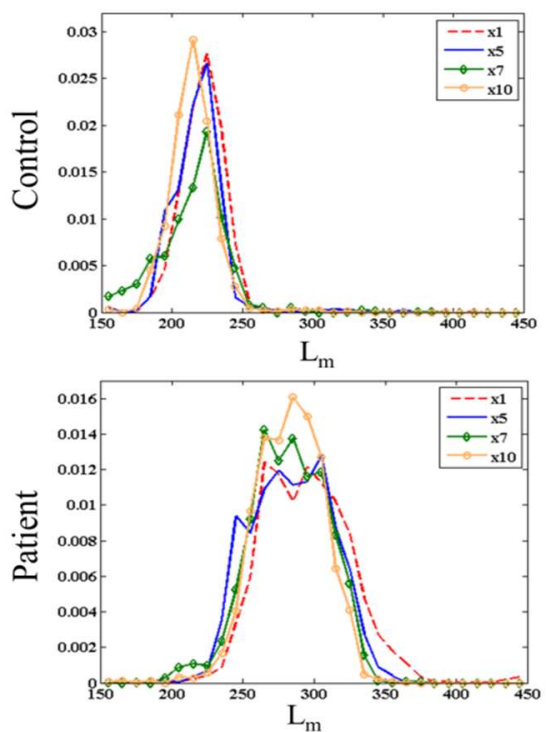

TV
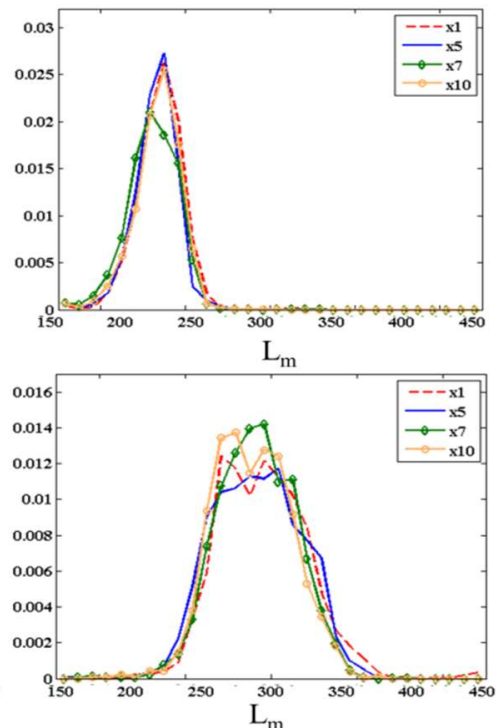

SIDER
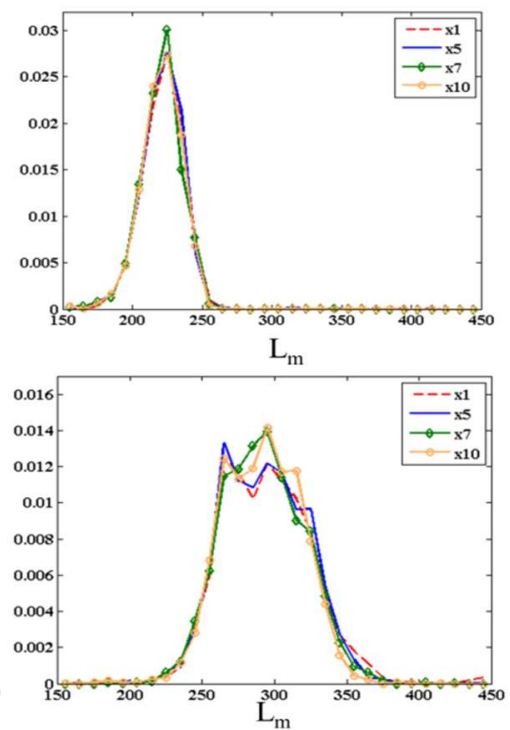

Fig. 5. Histograms of images of mean alveolar length for the reconstruction algorithms and acceleration factors for a control (top) and a patient (bottom).

2 Videos of results for all data sets are available from

https://github.com/HGGM-LIM/compressed-sensing-diffusion-lung-MRI.

(C) 20xx IEEE. Personal use of this material is permitted. Permission from IEEE must be obtained for all other uses, in any current or future media, including reprinting/republishing this material for advertising or promotional purposes, creating new collective works, for resale or redistribution to servers or lists, or reuse of any copyrighted component of this work in other works. DOI 10.1109/TMI.2017.2765281 
This article has been accepted for publication in a future issue of this journal, but has not been fully edited. Content may change prior to final publication. Citation information: DOI 10.1109/TMI.2017.2765281, IEEE Transactions on Medical Imaging

SIDER still preserved image quality with small errors in the highest acceleration factors. Image quality was preserved for all estimated maps at x10.

accelerations, although there were small deviations from the
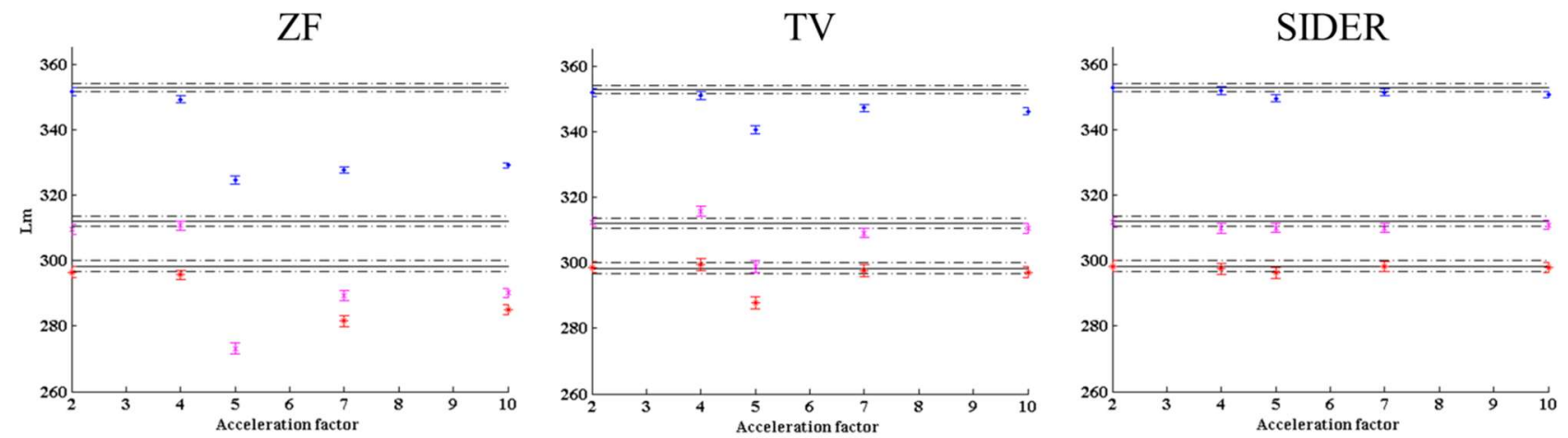

Fig. 6. Mean and SE of mean alveolar length across one slice vs. the acceleration factor for three different patients. Solid and dashed lines represent mean and SE, respectively, for fully sampled data.

Fig. 5 shows the histograms of estimated MAD from images reconstructed with the algorithms for a control and patient dataset. ZF and TV led to larger errors as the acceleration increased, becoming very noticeable above $x 7$. In contrast, SIDER presented histograms close to the target for accelerations up to $\times 10$. fully sampled map for an acceleration factor of x 10 .

Errors for the estimation of the mean and the histogram of $L_{m}$ are shown in Fig. 8. SIDER provides a very accurate estimation of the mean value of $\mathrm{L}_{\mathrm{m}}$, with errors below $1 \%$ for all accelerations. These errors were comparable to the experimental error (dotted line). An estimation of the histogram led to a value that was larger than the mean value. The cross- $\mathbf{x} 1$
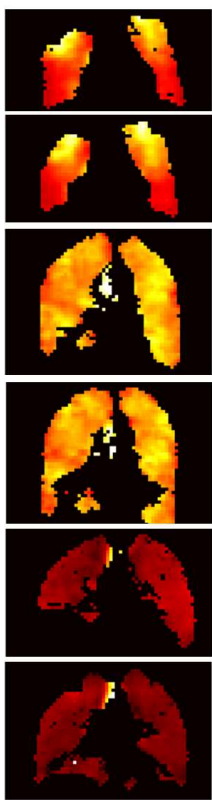

x5
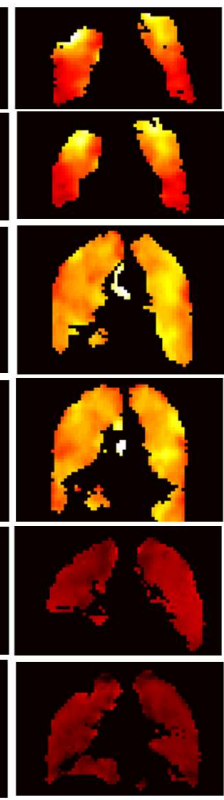

$\mathbf{x} 7$
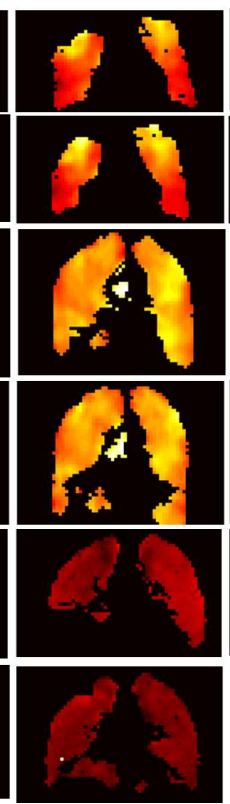

$\mathbf{x 1 0}$
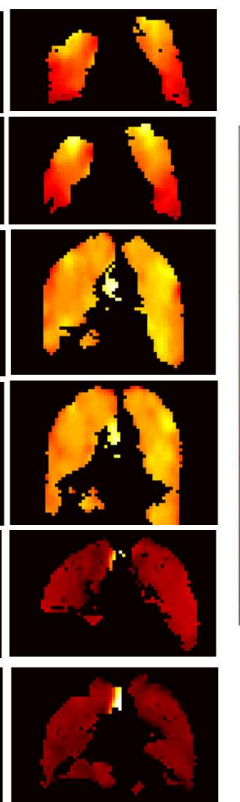

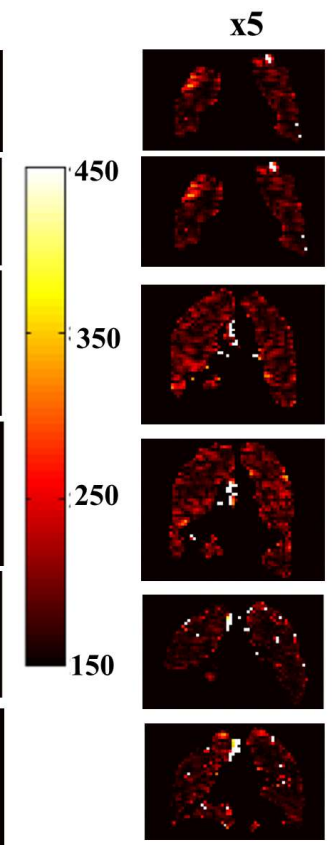

$\mathbf{x} 7$
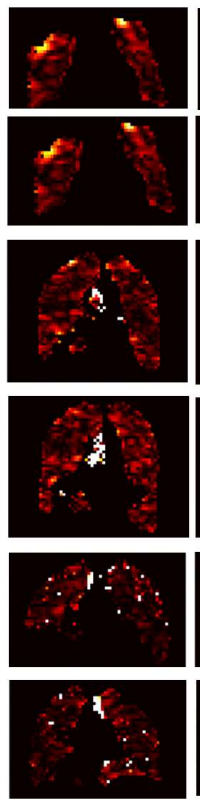

$\mathbf{x 1 0}$
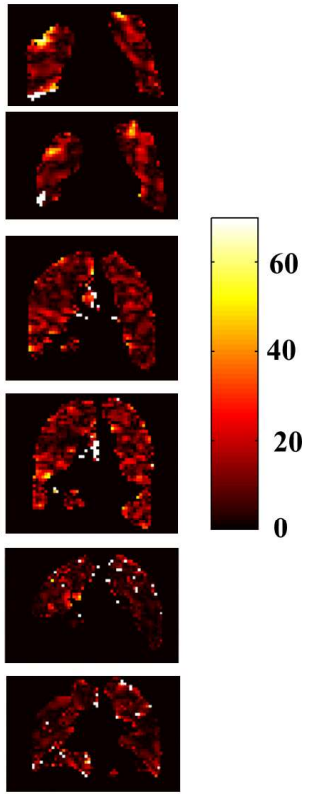

Fig. 7. One slice of estimated mean alveolar dimensions (left) and image differences (right) with SIDER for four patient and two control datasets.

Fig. 6 shows the mean and SE of MAD across one slice for three patients. For accelerations larger than five, errors by ZF and TV were larger than differences between datasets. In contrast, SIDER led to small errors for all acceleration factors.

\section{Analysis of the acceleration factor in retrospective data}

Fig. 7 shows a slice of the map of estimated MAD and image differences obtained by the SIDER method for the remaining datasets (two controls and four patient data sets) and for the correlation error for $\mathrm{x} 7$ was $2 \%$, which was similar to the experimental error. Higher accelerations (x10) led to larger errors.

Fig 9. shows histograms of the estimated MAD obtained from fully sampled data and using the SIDER method for acceleration factor $\mathrm{x} 7$ in all datasets. Histograms were very close to those of fully sampled data, and errors derived from the undersampling were much smaller than the intragroup differences between the control and patient datasets. 
This article has been accepted for publication in a future issue of this journal, but has not been fully edited. Content may change prior to final publication. Citation information: DOI 10.1109/TMI.2017.2765281, IEEE Transactions on Medical Imaging

Fig. 10 shows that the proposed method can be used to differentiate between controls and COPD patients based on the mean and FWHM of $L_{m}(\mathrm{p}<0.01)$. There were no significant differences between the proposed method and the complete data prior information. The proposed method, SIDER, was validated on both control and COPD patient data $(\mathrm{n}=8)$. Using retrospective undersampling, we found that accelerations of at least $\mathrm{x} 7$ are achievable with a negligible effect on the estimates
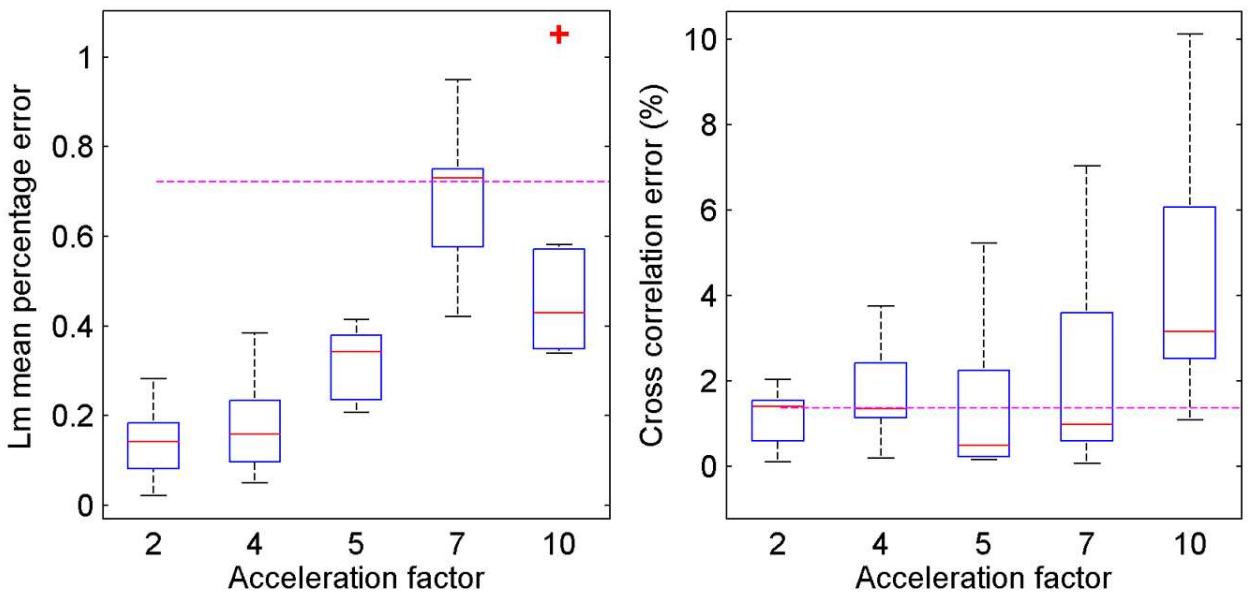

Fig 8. Left: Percentage error of the estimated average of the mean alveolar dimensions $\left(\mathrm{L}_{\mathrm{m}}\right)$ versus the acceleration factor. The mean and SD values shown were computed across all datasets. The dotted line represents the experimental error, which was estimated from two patients who had two acquisitions acquired at different sessions. Right: Percentage error of the estimation of the histogram of $\mathrm{L}_{\mathrm{m}}$. The estimation of the histogram is measured by the cross-correlation between the histogram for each acceleration factor and the histogram for the complete data. The error is computed with respect to the autocorrelation for the complete data.

for these metrics $(\mathrm{p}<0.01)$.

\section{Computation time}

The code was implemented in MATLAB on a Windows $\mathrm{x} 1$

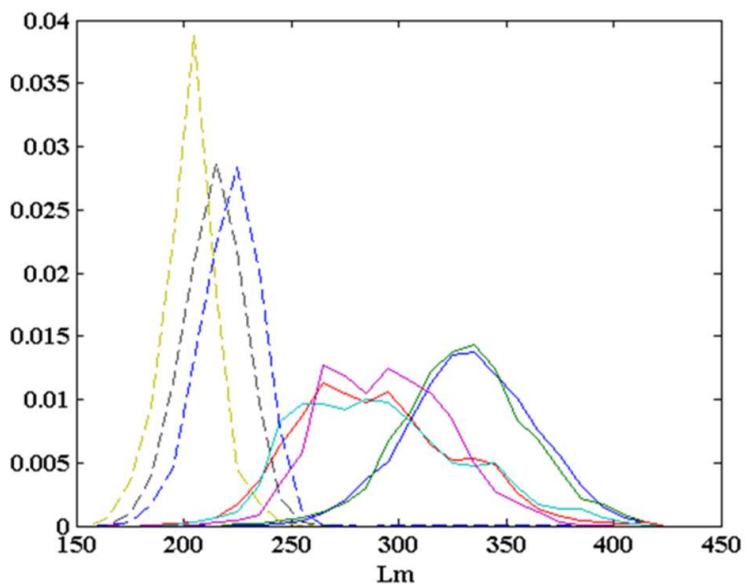

of ventilation images and estimated MAD maps. This acceleration factor (x7) is very relevant, as it could be used to increase the resolution by two-fold and simultaneously in the $\mathrm{x}$ $y$ plane, in the number of slices and in the number of b-values. Such an approach would not only increase the resolution and

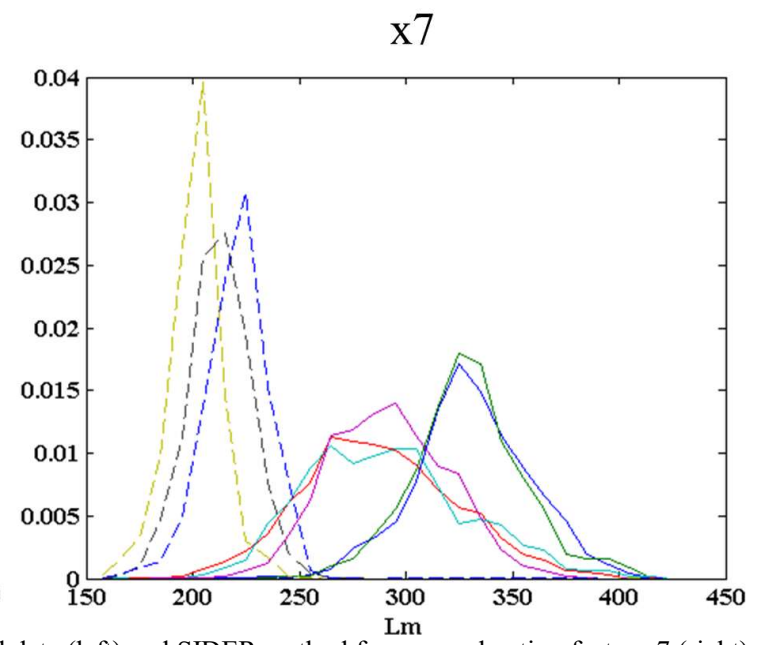

Fig. 9. Histograms of estimated mean alveolar dimensions for fully sampled data (left) and SIDER method for an acceleration factor x7 (right). Controls are depicted using dashed lines and COPD patients with solid lines.

computer with a 64-bit operating system, i7-3770, $3.40 \mathrm{GHz}$ CPU and 16 GB RAM. SIDER took $28 \mathrm{~s}$ to reconstruct all ventilation images (for 5 values of $b$ ) for one slice. We used a straightforward parallelization of SIDER over the five slices to reduce computation time.

\section{DISCUSSION}

We proposed and validated a novel CS method that incorporates a model of the signal decay into the reconstruction method as volume coverage, but also improve the accuracy of estimated microstructural dimensions and may enable the use of models with a larger number of parameters.

The acceleration factor depends on the criterion chosen to decide whether a result is acceptable. Adopting the experimental repeatability error as an upper bound, SIDER accurately calculated the mean of $L_{m}$ up to x 10 and of the entire histogram up to $x 7$. Nevertheless, this criterion may be very restrictive, as the experimental repeatability is very high. In

(C) 20xx IEEE. Personal use of this material is permitted. Permission from IEEE must be obtained for all other uses, in any current or future media, including reprinting/republishing this material for advertising or promotional purposes, creating new collective works, for resale or redistribution to servers or lists, or reuse of any copyrighted component of this work in other works. DOI 10.1109/TMI.2017.2765281 
This article has been accepted for publication in a future issue of this journal, but has not been fully edited. Content may change prior to final publication. Citation information: DOI 10.1109/TMI.2017.2765281, IEEE Transactions on Medical Imaging

fact, we showed that MSE remained small and histograms were similar to those obtained from fully sampled data for acceleration factors up to $\mathrm{x} 10$. Image quality was preserved with small errors in the estimated maps at a factor $\mathrm{x} 10$. A more practical criterion would be the ability to differentiate between controls and patients. In this case, SIDER provided the same results for $\mathrm{x} 7$ as those provided by fully sampled data, in terms of mean and FWHM of $L_{m}$, which are used in clinical practice. For accelerations higher than $\mathrm{x} 10$, errors were larger and the number of encoding lines acquired was very small for the approximations have been adopted for modeling signal decay. First, we assumed a stretched exponential model (3) with global parameters $\overline{\mathrm{D}}$ and $\bar{\alpha}$ averaged across the whole volume. Second, model parameters were estimated from a previously reconstructed image, which we chose as that provided by the TV method. We performed tests to assess these limitations. We compared the use of the global parameters obtained from TV and from SIDER (by running SIDER twice), although no significant improvements were observed. This could be explained by the fact that average parameters provided by TV
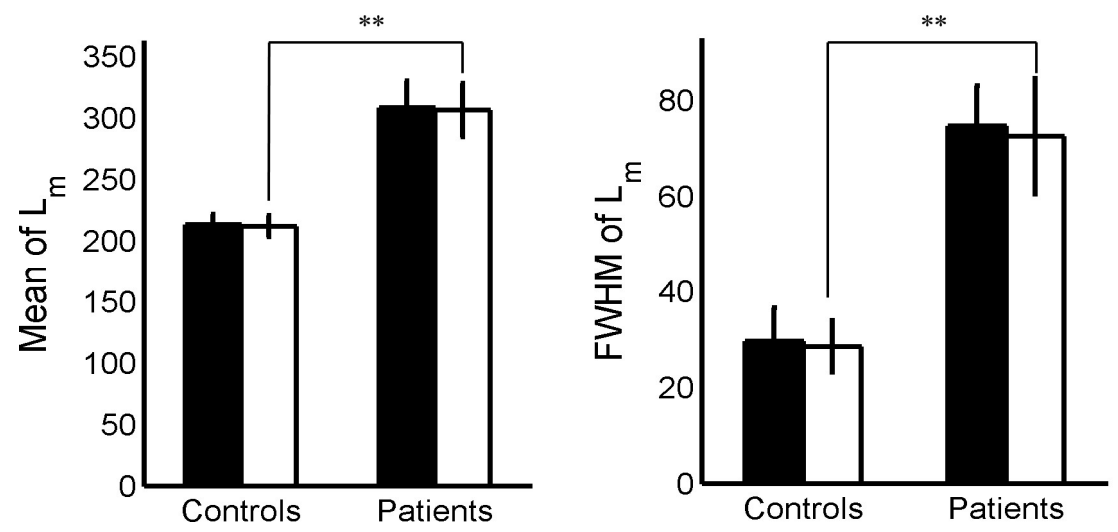

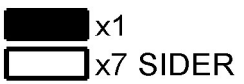

$* * \mathrm{p}<0.01$

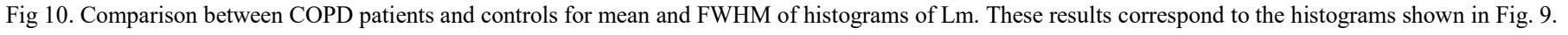

present resolution (images of $64 \times 64$ ), which was not considered acceptable. An acceleration factor of $\mathrm{x} 7$, which would enable doubling of the spatial resolution, number of slices and the number of b-values, presented negligible errors.

Previous CS implementations achieved lower acceleration factors: $x 2$ using spatial TV in 2D [14], x3 using spatial TV in $2 \mathrm{D}$ with prior knowledge of a proton image acquired during the same breath-hold [15], and $x 3$ using TV in 3D [16]. The acceleration factors achieved in this study, from $\mathrm{x} 7$ to $\mathrm{x} 10$, are superior because of the exploitation of undersampling along the b-dimension and the use of a reconstruction model that incorporates prior knowledge of signal decay. SIDER could also be extended to the 3D case as in [16], potentially achieving even higher accelerations.

In this study, we aimed to exploit sparsity not only in the spatial domain, but also across the b-dimension, by both modeling the signal decay and randomizing the acquisition along the b-direction. The proposed SIDER method is a combination of spatial TV and a functional that models signal decay. Given that SIDER and TV are both solved using the split Bregman formulation, the only difference between these two methodologies lies in the exploitation of the b-dimension. TV has been chosen as spatial regularization, which had been previously proposed for hyperpolarized gas MRI [14]-[16]. Other functionals for spatial regularization, such as total generalized variation (TGV) [41], [42], could lead to results that are superior to those of TV. Nevertheless, TGV or different spatial regularization could be included in the proposed methodology by replacing the TV functional.

Our study is subject to several limitations. Various were sufficient to guide the reconstruction algorithm. We also found that using zero filling instead of TV led to large errors for high acceleration factors. Thus, zero filling could not provide a good estimate of average parameters for the stretched exponential model. A more simplified assumption could be to model the signal decay for consecutive values of $b$ with an exponential model, $M u_{j}=u_{j}-u_{j-1} \exp \left[-\bar{D}\left(b_{j}-b_{j-1}\right)\right]$. In this case, using zero filling to estimate $\bar{D}$ across the whole volume led to reasonably good results. This approximation is faster and simpler, as it requires a less accurate estimation of $\bar{D}$, although it provided slightly worse results for some values of $b$ than the proposed model. A plausible improvement in the method proposed could be to model the parameters of the stretched model ( $D$ and $\alpha$ ) on a pixel-by-pixel basis (instead of average parameters) and to include them as unknowns in the reconstruction procedure. This method could use the reconstructed image to update the signal decay model in an iterative fashion and iterate until convergence. Another improvement could be to rewrite the reconstruction problem in terms of $D$ and $\alpha$ or in terms of $L_{m}$. However, such an approach lies beyond the scope of the present study.

Another limitation is that we validated the method by retrospectively undersampling control and patient data. However, implementation of the proposed CS sequence in an MR scanner will be validated in future studies. In addition, the potential gain in microstructure information is promising but requires a dedicated study.

In future studies, this method will be extended to the acquisition of 3D diffusion data, where higher accelerations can 
This article has been accepted for publication in a future issue of this journal, but has not been fully edited. Content may change prior to final publication. Citation information: DOI 10.1109/TMI.2017.2765281, IEEE Transactions on Medical Imaging

be achieved through undersampling over 3 directions (e.g. 2 spatial directions plus b-value), and to other hyperpolarized gas MR applications, where a model of the signal behavior has been assessed (e.g., $\mathrm{pO}_{2}$ mapping [7]). Our method is not restricted to hyperpolarized gas imaging, but could also be used in other diffusion MRI applications and in metabolic imaging based on hyperpolarized ${ }^{13} \mathrm{C}$.

In conclusion, we validated a novel CS method for lung MRI imaging and achieved high acceleration factors that can be used to increase the amount of data acquired during breath-hold. This methodology is expected to improve the accuracy of estimated lung microstructure data and widen the possibilities of studying lung diseases with MRI.

\section{ACKNOWLEDGMENT}

The MRI diffusion data used in this work were kindly made available by Prof. Jim M. Wild (POLARIS, Academic Unit of Radiology, University of Sheffield, UK).

\section{REFERENCES}

[1] M. Salerno, T. A. Altes, J. P. Mugler, M. Nakatsu, H. Hatabu, and E. E. de Lange, "Hyperpolarized noble gas MR imaging of the lung: potential clinical applications.," Eur. J. Radiol., vol. 40, no. 1, pp. 33-44, Oct. 2001.

[2] J. E. Roos, H. P. McAdams, S. S. Kaushik, and B. Driehuys, "Hyperpolarized Gas MR Imaging," Magn. Reson. Imaging Clin. N. Am., vol. 23, no. 2, pp. 217-229, May 2015.

[3] A. J. Deninger et al., "Quantitative measurement of regional lung ventilation using ${ }^{3}$ He MRI," Magn. Reson. Med., vol. 48, no. 2, pp. 223-232, Aug. 2002.

[4] F. C. Horn, M. H. Deppe, H. Marshall, J. Parra-Robles, and J. M. Wild, "Quantification of regional fractional ventilation in human subjects by measurement of hyperpolarized $3 \mathrm{He}$ washout with 2D and 3D MRI.," J. Appl. Physiol., vol. 116, no. 2, pp. 129-39, Jan. 2014.

[5] Y. Wu et al., "Accelerated MR diffusion tensor imaging using distributed t sensing," Magn. Reson. Med., vol. 71, no. 2, pp. $763-$ 772, Feb. 2014.

[6] A. J. Deninger et al., "Quantification of regional intrapulmonary oxygen partial pressure evolution during apnea by (3)He MRI.," $J$. Magn. Reson., vol. 141, no. 2, pp. 207-16, Dec. 1999.

[7] H. Marshall, J. Parra-Robles, M. H. Deppe, D. A. Lipson, R. Lawson, and J. M. Wild, "(3)He pO2 mapping is limited by delayed-ventilation and diffusion in chronic obstructive pulmonary disease.," Magn. Reson. Med., vol. 71, no. 3, pp. 1172-8, Mar. 2014.

[8] M. H. Deppe, J. Parra-Robles, S. Ajraoui, and J. M. Wild, "Combined measurement of pulmonary inert gas washout and regional ventilation heterogeneity by MR of a single dose of hyperpolarized 3He.," Magn. Reson. Med., vol. 65, no. 4, pp. 107583, Apr. 2011.

[9] T. G. Walker and W. Happer, "Spin-exchange optical pumping of noble-gas nuclei," Rev. Mod. Phys., vol. 69, no. 2, pp. 629-642, Apr. 1997.

[10] S. R. Parnell, M. H. Deppe, J. Parra-Robles, and J. M. Wild, "Enhancement of X129e polarization by off-resonant spin exchange optical pumping," J. Appl. Phys., vol. 108, no. 6, p. 64908, Sep. 2010.

[11] J. M. Wild et al., "Simultaneous Imaging of Lung Structure and Function with Triple-Nuclear Hybrid MR Imaging," Radiology, vol. 267, no. 1, pp. 251-255, Apr. 2013.

[12] R. F. Lee, G. Johnson, R. I. Grossman, B. Stoeckel, R. Trampel, and G. McGuinness, "Advantages of parallel imaging in conjunction with hyperpolarized helium--a new approach to MRI of the lung.," Magn. Reson. Med., vol. 55, no. 5, pp. 1132-41, May 2006. morphometry with accelerated hyperpolarized ${ }^{3} \mathrm{He}$ diffusion MRI: A preliminary study," Magn. Reson. Med., vol. 73, no. 4, pp. 1609 1614, Apr. 2015.

[14] S. Ajraoui, K. J. Lee, M. H. Deppe, S. R. Parnell, J. Parra-Robles, and J. M. Wild, "Compressed sensing in hyperpolarized $3 \mathrm{He}$ lung MRI.," Magn. Reson. Med., vol. 63, no. 4, pp. 1059-69, Apr. 2010.

[15] S. Ajraoui, J. Parra-Robles, and J. M. Wild, "Incorporation of prior knowledge in compressed sensing for faster acquisition of hyperpolarized gas images.," Magn. Reson. Med., vol. 69, no. 2, pp. 360-9, Feb. 2013.

[16] H.-F. Chan, N. J. Stewart, J. Parra-Robles, G. J. Collier, and J. M. Wild, "Whole lung morphometry with 3D multiple b-value hyperpolarized gas MRI and compressed sensing.," Magn. Reson. Med., vol. 77, no. 5, pp. 1916-1925, May 2017.

[17] A. J. Swift et al., "Emphysematous changes and normal variation in smokers and COPD patients using diffusion 3He MRI.," Eur. J. Radiol., vol. 54, no. 3, pp. 352-8, Jun. 2005.

[18] J. Parra-Robles and J. M. Wild, "The influence of lung airways branching structure and diffusion time on measurements and models of short-range $3 \mathrm{He}$ gas MR diffusion," J. Magn. Reson., vol. 225, pp. 102-113, Dec. 2012.

[19] J. Parra-Robles, S. Ajraoui, H. Marshall, M. H. Deppe, X. Xu, and J. M. Wild, "The influence of field strength on the apparent diffusion coefficient of $3 \mathrm{He}$ gas in human lungs.," Magn. Reson. Med., vol. 67, no. 2, pp. 322-5, Feb. 2012.

[20] O. Michailovich, Y. Rathi, and S. Dolui, "Spatially regularized compressed sensing for high angular resolution diffusion imaging.," IEEE Trans. Med. Imaging, vol. 30, no. 5, pp. 1100-15, May 2011.

[21] B. A. Landman, J. A. Bogovic, H. Wan, F. E. Z. ElShahaby, P.-L. Bazin, and J. L. Prince, "Resolution of crossing fibers with constrained compressed sensing using diffusion tensor MRI," Neuroimage, vol. 59, no. 3, pp. 2175-2186, Feb. 2012.

[22] L. Ning et al., "A joint compressed-sensing and super-resolution approach for very high-resolution diffusion imaging," Neuroimage, vol. 125, pp. 386-400, Jan. 2016.

[23] B. Bilgic, K. Setsompop, J. Cohen-Adad, A. Yendiki, L. L. Wald, and E. Adalsteinsson, "Accelerated diffusion spectrum imaging with compressed sensing using adaptive dictionaries," Magn. Reson. Med., vol. 68, no. 6, pp. 1747-1754, Dec. 2012.

[24] M. I. Menzel et al., "Accelerated diffusion spectrum imaging in the human brain using compressed sensing," Magn. Reson. Med., vol. 66, no. 5, pp. 1226-1233, Nov. 2011.

[25] M. Paquette, S. Merlet, G. Gilbert, R. Deriche, and M. Descoteaux, "Comparison of sampling strategies and sparsifying transforms to improve compressed sensing diffusion spectrum imaging," Magn. Reson. Med., vol. 73, no. 1, pp. 401-416, Jan. 2015.

[26] S. G. Lingala, Y. Hu, E. DiBella, and M. Jacob, "Accelerated dynamic MRI exploiting sparsity and low-rank structure: k-t SLR.," IEEE Trans. Med. Imaging, vol. 30, no. 5, pp. 1042-54, May 2011.

[27] P. Montesinos, J. F. P. J. Abascal, L. Cussõ, J. J. Vaquero, and M. Desco, "Application of the compressed sensing technique to selfgated cardiac cine sequences in small animals," Magn. Reson. Med., vol. 72 , no. $2,2014$.

[28] J. F. P. J. Abascal, P. Montesinos, E. Marinetto, J. Pascau, and M. Desco, "Comparison of Total Variation with a Motion Estimation Based Compressed Sensing Approach for Self-Gated Cardiac Cine MRI in Small Animal Studies," PLoS One, vol. 9, no. 10, p. e110594, Oct. 2014.

[29] C. Chavarrías, J. F. P. J. Abascal, P. Montesinos, and M. Desco, "Exploitation of temporal redundancy in compressed sensing reconstruction of fMRI studies with a prior-based algorithm (PICCS)," Med. Phys., vol. 42, no. 7, pp. 3814-3821, Jun. 2015.

[30] M. Chiew, S. M. Smith, P. J. Koopmans, N. N. Graedel, T. Blumensath, and K. L. Miller, "k-t FASTER: Acceleration of functional MRI data acquisition using low rank constraints," Magn. Reson. Med., vol. 74, no. 2, pp. 353-364, Aug. 2015.

[31] J. Parra-Robles, H. Marshall, and J. M. Wild, "Characterization of 3 He Diffusion in Lungs using a Stretched Exponential Model," in Proc. Intl. Soc. Mag. Reson. Med. , 2013, p. 820.

[32] J. Parra-Robles, H. Marshall, R. A. Hartley, C. E. Brightling, and J. M. Wild, "Quantification of lung microstructure in asthma using a $3 \mathrm{He}$ fractional diffusion approach," in Proc. Intl. Soc. Mag. Reson. Med., 2014, p. 3529.

(C) 20xx IEEE. Personal use of this material is permitted. Permission from IEEE must be obtained for all other uses, in any current or future media, including reprinting/republishing this material for advertising or promotional purposes, creating new collective works, for resale or redistribution to servers or lists, or reuse of any copyrighted component of this work in other works. DOI 10.1109/TMI.2017.2765281 
This article has been accepted for publication in a future issue of this journal, but has not been fully edited. Content may change prior to final publication. Citation information: DOI 10.1109/TMI.2017.2765281, IEEE Transactions on Medical Imaging

[33] S. Osher, M. Burger, D. Goldfarb, J. Xu, and W. Yin, “An Iterative Regularization Method for Total Variation-Based Image Restoration," Multiscale Model. Simul., vol. 4, no. 2, pp. 460-489, Jan. 2005.

[34] T. Goldstein and S. Osher, "The Split Bregman Method for L1Regularized Problems," SIAM J. Imaging Sci., vol. 2, no. 2, pp. 323-343, Jan. 2009.

[35] M. Lustig, D. Donoho, and J. M. Pauly, "Sparse MRI: The application of compressed sensing for rapid MR imaging," Magn. Reson. Med., vol. 58, no. 6, pp. 1182-1195, Dec. 2007.

[36] P. Montesinos, J. F. P. J. Abascal, L. Cussó, J. J. Vaquero, and M. Desco, "Application of the compressed sensing technique to selfgated cardiac cine sequences in small animals," Magn. Reson. Med., vol. 72, no. 2, pp. 369-380, Aug. 2014.

[37] J. F. P.-J. Abascal et al., "Fluorescence diffuse optical tomography using the split Bregman method," Med. Phys., vol. 38, no. 11, 2011.

[38] J. F. P. J. Abascal, M. Abella, E. Marinetto, J. Pascau, M. Desco, and K. Ang, "A Novel Prior- and Motion-Based Compressed Sensing Method for Small-Animal Respiratory Gated CT," PLoS One, vol. 11, no. 3, p. e0149841, Mar. 2016.

[39] P. C. Hansen and D. P. O'Leary, "The Use of the L-Curve in the Regularization of Discrete Ill-Posed Problems," SIAM J. Sci. Comput., vol. 14, no. 6, pp. 1487-1503, Nov. 1993.

[40] J.-F. P. J. Abascal, S. R. Arridge, R. H. Bayford, and D. S. Holder, "Comparison of methods for optimal choice of the regularization parameter for linear electrical impedance tomography of brain function," Physiol. Meas., vol. 29, no. 11, 2008.

[41] K. Bredies, K. Kunisch, and T. Pock, "Total Generalized Variation," SIAM J. Imaging Sci., vol. 3, no. 3, pp. 492-526, Jan. 2010.

[42] F. Knoll, K. Bredies, T. Pock, and R. Stollberger, "Second order total generalized variation (TGV) for MRI," Magn. Reson. Med., vol. 65 , no. 2 , pp. 480-491, Feb. 2011.

(C) 20xx IEEE. Personal use of this material is permitted. Permission from IEEE must be obtained for all other uses, in any current or future media, including reprinting/republishing this material for advertising or promotional purposes, creating new collective works, for resale or redistribution to servers or lists, or reuse of any copyrighted component of this work in other works. DOI 10.1109/TMI.2017.2765281 